\title{
Graft Transmissible Agents Affect Membrane Fatty Acid Saturation during Dormancy Release in Peach
}

\author{
Philip G. Gibson ${ }^{1}$ \\ Environmental Horticulture, Gwinnett Technical College, Lawrenceville, GA 30046 \\ Gregory L. Reighard \\ Department of Horticulture, Clemson University, Clemson, SC 29634
}

Gary L. Powell

Department of Biological Sciences, Clemson University, Clemson, SC 29634

Thomas C. Jenkins

Department of Animal and Veterinary Sciences, Clemson, SC 29634

\begin{abstract}
AdDitional IndeX wORDs. Prunus persica, viroid, desaturase, linoleic, linolenic, inoculation
Abstract. Peach [Prunus persica (L.) Batsch (Peach Group)] trees infected with peach latent mosaic viroid (PLMVd) have been associated with phenological changes including delay in bloom, reduced shoot vigor, and early autumn defoliation. In order to further characterize the changes occurring in trees inoculated with PLMVd, total fatty acid content was measured for floral buds during release from dormancy in 'Coronet' peach trees. Palmitic (16:0), stearic (18:0), oleic (18:1), linoleic (18:2), and linolenic (18:3) acids were the major fatty acids in dormant and releasing peach buds of both control and PLMVd-inoculated (VI) trees. The degree of unsaturation increased immediately following release from dormancy in both the control and VI trees. However, desaturation of linoleic acid to linolenic acid was significantly inhibited in VI trees, which was accompanied by a concomitant delay in the resumption of growth. The disparity between the control and VI trees in the progression of increased fatty acid unsaturation continued through petal fall. The presence of PLMVd in 'Coronet' peach trees slowed membrane fatty acid desaturation during release from dormancy and suggested that metabolic pathways involving fatty acid desaturation were linked to the delayed phenology of the VI trees.
\end{abstract}

Plant response to temperature patterns in the environment requires an internal mechanism that is responsive to temperature fluctuations, but includes a variety of control processes which prevent inappropriate responses to minor fluctuations. The resumption of growth following dormancy is accompanied by a change in membrane composition permitting increased permeability of solutes and water (Brockerhof, 1974; Goad, 1983; Grunwald, 1975; Oldfield and Chapman, 1972). The degree of unsaturation in fatty acids of membrane lipids is a cellular response to changes in temperature which facilitates maintenance of an optimal degree of membrane fluidity (Cossins, 1983; Harris and James, 1968; Lynch and Steponkus, 1987; Thompson, 1979; Yoshida, 1984). Membrane permeability is partially controlled by fatty acid composition, which can limit or promote cellular metabolic activity (Sikorska and Farkus, 1982). Desaturase enzyme activity is correlated with the membrane changes taking place during endodormancy (Faust and Wang, 1993). The association between membrane fatty acid desaturation and a plant's adaptation to environmental temperature changes is well established (Murakami, et al., 2000; Yoshida, 1984; Wang and Faust, 1988). Specifically, Wang and Faust (1990) found an accumulation of linolenate as apple buds released from dormancy. In peach, the

\footnotetext{
Received for publication 3 Jan. 2004. Accepted for publication 16 Apr. 2004. This material is based upon work supported by the CSREES/USDA, under project number SC-1700005 and was financially supported by the South Carolina Peach Council. We thank David Ouellette, Kathy Brock, Evanne Thies, and William Newall, Jr. for technical assistance.

${ }^{1}$ Current address: Gwinnett Technical College, 5150 Sugarloaf Parkway, Lawrenceville, GA 30043; phone: 770-962-7580 xt 282; fax: 770-962-7985.

E-mail address: PGibson@gwinnett.tec.ga.us
}

relative proportions of linolenate and linoleate parallel the effects of temperature on dormancy and resumption of growth (Erez et al., 1997). Both of these studies found that linoleate accumulates during chilling followed by an accumulation of linolenate during exposure to temperatures favorable to bud development after rest completion. The rise in linolenate concentration upon resumption of growth is accompanied by a reduction in linoleate concentrations. This can be explained by the activity of specific desaturase enzymes, which insert the third double bond into the linoleate substrate.

Peach latent mosaic viroid (PLMVd) is present as a graft transmissible agent in the peach cultivar 'Ta Tao 5' (Diener, 1987). Phenological effects attributed to PLMVd are graft transmissible and include a delay in flowering and fruit maturity, increased fruit firmness, higher yield efficiency, earlier fall defoliation, and reduced tree vigor (Gibson and Reighard, 2002; Reighard, 1998). The objective of this study was to investigate the seasonal change during dormancy of fatty acids in cell membranes of peach floral buds infected with PLMVd.

\section{Materials and Methods}

Plant material. Floral buds were collected from 'Coronet' peach trees planted Jan. 1996 as a high-density, Y-trained orchard system. The 'Coronet' trees were in four rows that were divided into a completely randomized block design consisting of six blocks of six trees in each row. Half the blocks were randomly selected for PLMVd inoculation with 'Ta Tao 5' peach vegetative chip buds in Aug. 1997. The trees were maintained under normal, commercial production cultural practices. The presence of PLMVd was confirmed in the inoculated 'Coronet' trees (VI) 

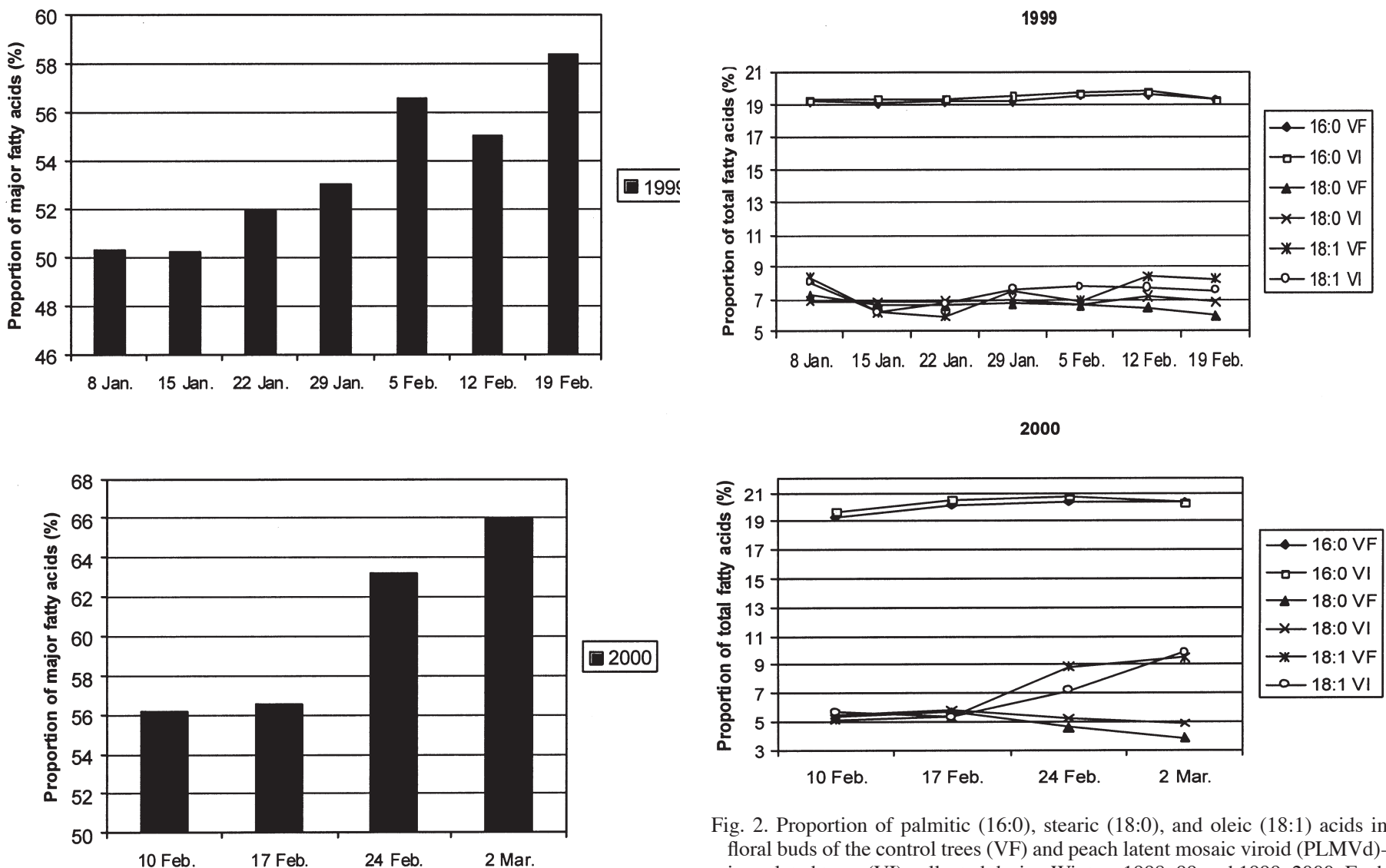

Fig. 2. Proportion of palmitic (16:0), stearic (18:0), and oleic (18:1) acids in floral buds of the control trees (VF) and peach latent mosaic viroid (PLMVd)inoculated trees (VI) collected during Winters 1998-99 and 1999-2000. Each data point represents the average of eight samples. For each sample date, there were no significant differences in fatty acid content between the control and VI trees $(t$ test $P=0.05)$

Proportion of the top five fatty acids (FA); consisting of palmitic stearic (18:0), oleic (18:1), linoleic (18:2), and linolenic (18:3) acids found in floral buds of peach trees utilized in this study during Winters 1998-99 and 1999-2000. Graph represents pooled data from all floral buds collected on each date.

used in this study by dot blot cRNA probe hybridization (Gibson et al., 2001b; Shamloul, 1995). Floral buds were collected from both of the two outside trees (tree 1 and 6) in each block during Jan., Feb., and Mar. 1999 and 2000. Buds from each outside tree of each VI or control block in rows one to four were pooled and duplicated to yield a total of eight VI tree samples and eight control tree samples for each collection date. Buds were stored at $-80{ }^{\circ} \mathrm{C}$ until the samples were processed in each year. Frozen buds were ground in a coffee grinder, weighed and processed immediately for fatty acid analysis.

FatTy ACID ANaLYSIS. Fatty acids were methylated to fatty acyl methyl esters (FAME) using a direct transesterification procedure (Sukhija and Palmquist, 1988). Samples were weighed to $500 \mathrm{mg}$ and added to a $15 \times 150-\mathrm{mm}$ culture tube with screw cap and Teflon liner. Heptadecanoic acid (17:0) was added as an internal standard $\left(500 \mathrm{mg} \cdot \mathrm{mL}^{-1}\right.$ benzene) to each tube. Next, $3 \mathrm{~mL}$ of $5 \% \mathrm{v} / \mathrm{v}$ methanolic $\mathrm{HCl}$ was added, tubes were capped tightly and vortexed. Samples were incubated in a $70{ }^{\circ} \mathrm{C}$ water bath for $2 \mathrm{~h}$. Tubes were cooled to room temperature, $7.5 \mathrm{~mL}$ of $6 \% \mathrm{w} / \mathrm{v} \mathrm{K}_{2} \mathrm{CO}_{3}$ was added, $1 \mathrm{~mL}$ hexane, capped and vortexed. After centrifugation at $10,000 g_{\mathrm{n}}$ for $5 \mathrm{~min}$, the organic layer was transferred to a gas chromatograph sampling vial with a Teflon lined cap. FAMEs were analyzed by flame ionization detection gas chromatography (FID-GC) using a Supelco P-2380 (Supelco, Bellefonte, Pa.) fused silica capillary column $30 \mathrm{~m} \times 0.25 \mathrm{~mm} \times$ $0.2-\mu \mathrm{m}$ film thickness. The HP5890A gas chromatograph oven temperature was ramped from at $140{ }^{\circ} \mathrm{C}$ for $3 \mathrm{~min}$. to $220^{\circ} \mathrm{C}$ at

$3.7^{\circ} \mathrm{C} / \mathrm{m}$ and held for $20 \mathrm{~min}$. Helium carrier rate was $20 \mathrm{~cm} \cdot \mathrm{s}^{-1}$ at $150^{\circ} \mathrm{C}$ with a flame ionization detector temperature of $260^{\circ} \mathrm{C}$. The injector was split $100: 1$ at $250^{\circ} \mathrm{C}$ with column head pressure of $87 \mathrm{kPa}$. The flow rate was $35.0 \mathrm{He}$ at tank pressure $276 \mathrm{kPa}$. Individual FAMEs were identified by comparing their retention times with authentic standards (Supelco). The FID FAME identification results were confirmed by mass spectroscopy.

\section{Results and Discussion}

Palmitic (16:0), stearic (18:0), oleic (18:1), linoleic (18:2), and linolenic (18:3) acids were the major fatty acids in dormant and releasing peach buds of both the control and PLMVd-inoculated 'Coronet' (VI) trees (Fig. 1). Concentrations of palmitic, stearic, and oleic acids remained relatively constant throughout the experiment (Fig. 2). The increase in linolenic acid in the flower buds from the VI trees lagged significantly behind the control trees during release from dormancy in 1999 and 2000 (Fig. 3). A differential change to linolenic and linoleic acids was found between the VI and control trees (Fig. 4). The larger ratio of saturated [palmitic (16:0) and stearic (18:0)] to unsaturated fatty acids [oleic (18:1), linoleic (18:2), and linolenic (18:3)] in the VI trees indicated higher degree of unsaturation in the control trees (Fig. 5).

The presence of PLMVd did not change the chilling requirement of 'Coronet' peach (Gibson and Reighard, 2002). The response of peach blossoms to forcing conditions is dependent upon chilling accumulation and subsequent heat accumulation (Richardson et al., 1975). Breaking dormancy requires heat unit accumulation 


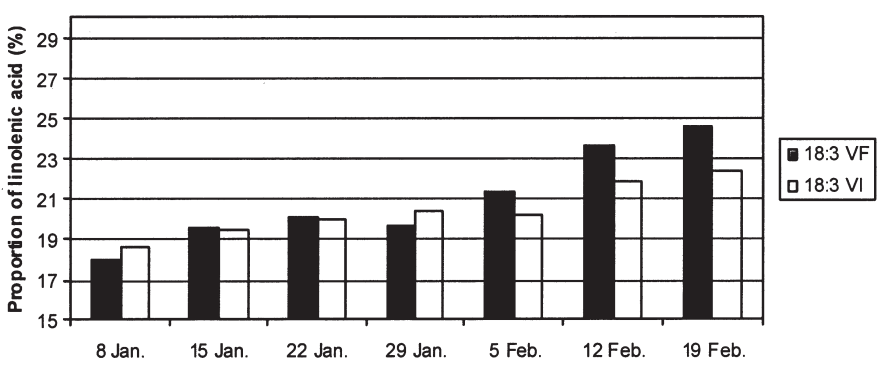

2000

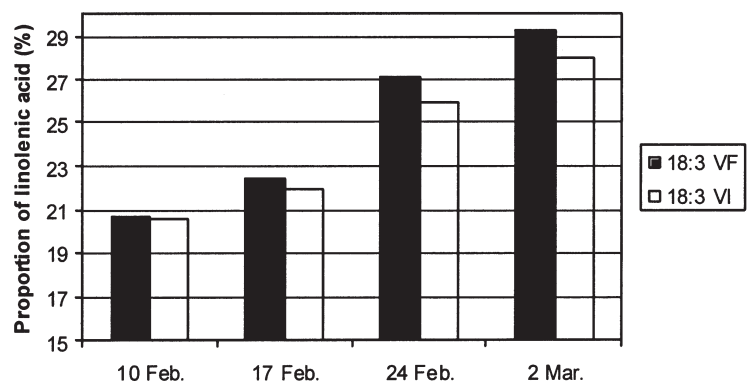

Fig. 3. Proportion of linolenic acid (18:3) in floral buds from control trees (VF) and peach latent mosaic viroid (PLMVd)-inoculated trees (VI) collected during the 1999 and 2000 release from dormancy. Each data point represents the average of eight samples. Differences in percent linolenic acid between the control and VI trees for sample dates after 5 Feb. 1999 and 17 Feb. 2000 were significant ( $t$ test $P=0.05$ ).

(Arnold, 1959) above a base temperature. Accumulation of heat units necessary to release buds is the growth-limiting factor during ecodormancy (Ashcroft et al., 1977; Richardson et al., 1975; Spiegel-Roy and Alston, 1979). Studies have shown that active growth or resumption of growth requires unsaturated fatty acids as constituents of membrane lipids (Erez et al., 1997). Chilling produces a membrane structure that is conducive to resumption of growth and corresponds to the accumulation of linoleic acid (Wang and Faust, 1990). Desaturation of linoleic acid to linolenic acid occurs at growth promoting warm temperatures. Therefore, budbreak is dependent upon the level of linolenic acid as a membrane lipid constituent and the energy available to drive the desaturase enzyme (Norman et al., 1991). ATPase activity and sugar availability during this process are critical because the desaturation of 18:2 to 18:3 requires a substantial amount of reducing power and active oxygen, in addition to detoxification of $\mathrm{H}_{2} \mathrm{O}_{2}$ to prevent inactivation of desaturase enzymes. High levels of glutathione have been closely associated with peach buds breaking dormancy, which may also be related to membrane desaturation (Siller-Cepeda et al., 1992a, 1992b). The initial level of linolenic acid in the VI trees was similar to that of the control trees, but the accumulation of linolenic acid was significantly reduced in the VI trees. Additionally, the degree of desaturation of fatty acids was suppressed in the VI trees. These differences may be the result of several physiological anomalies in trees inoculated with PLMVd.

The VI trees could have a reduction in the synthesis or activity of the desaturase enzyme, a lower energy reserve, and/or an inhibitory mechanism. The highest respiration rates measured in peach trees occurs in the early stages of vegetative budbreak following release from dormancy (Grossman and Dejong, 1994). Therefore, competition among metabolic processes for available

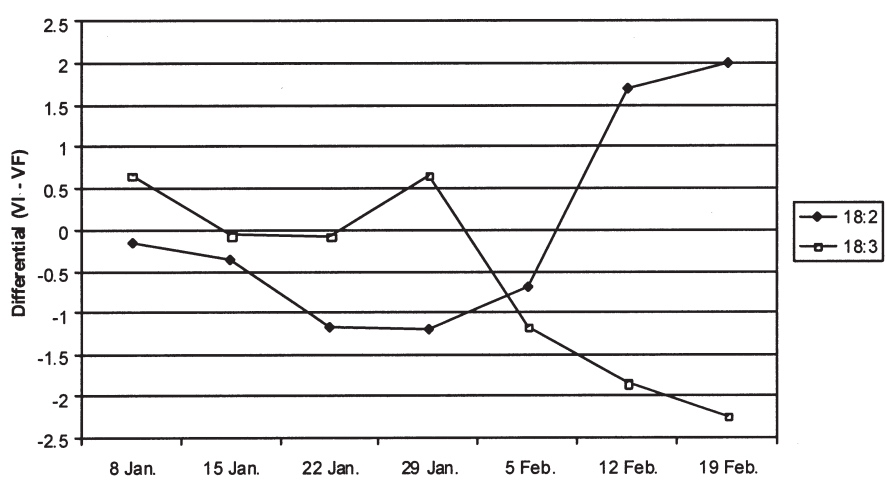

2000

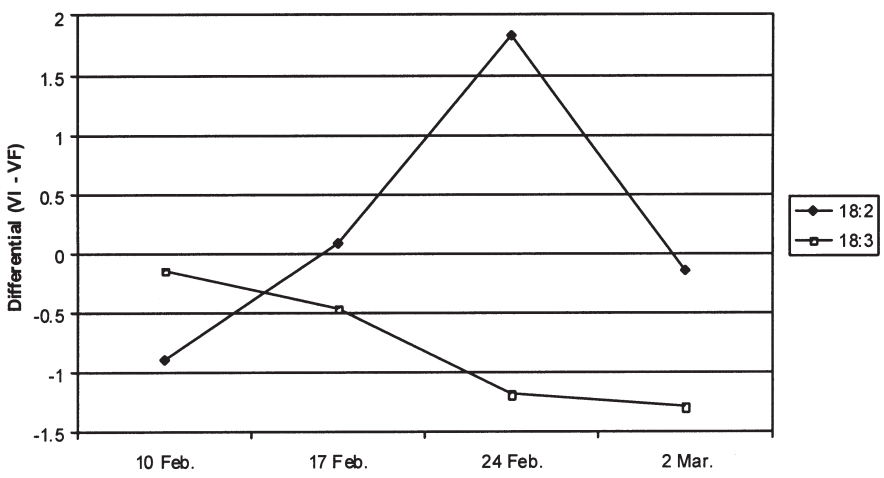

Fig. 4. Differential change in linolenic (18:3) and linoleic (18:2) acids during release from dormancy in 1999 and 2000. Values calculated by subtracting values for the control trees (VF) from those of the peach latent mosaic viroid (PLMVd)-inoculated trees (VI). Each data point represents the average of 8 samples. Differential changes in linolenic acid and linoleic acid for sample dates after 5 Feb. 1999 and 17 Feb. 2000 were significant ( $t$ test $P=0.05$ ).

energy is significant during release from dormancy. Evidence from field studies indicates significantly early fall defoliation of 5 to $6 \mathrm{~d}$ on VI trees (Gibson et al., 2001a), which could reduce carbohydrate reserves. Moreover, a higher heat accumulation is required for resumption of growth in the spring for VI trees (Gibson and Reighard, 2002). These findings, coupled with the fatty acid changes reported here, provide further evidence that the effect of PLMVd in peach may be the suppression of normal physiological processes, which result in a delay in the response to budbreak conditions upon completion of chilling. Further study of how these physiological processes are affected by PLMVd is needed to elucidate the mode of action of this viroid on peach phenology.

\section{Literature Cited}

Arnold, C.Y. 1959. The determination and significance of the base temperature in a linear heat unit system. Proc. Amer. Soc. Hort. Sci. 74:430-445.

Ashcroft, G.L., E.A. Richardson, and D.S. Schuyler. 1977. A statistical method of determining chill unit and growing degree hour requirements for deciduous fruit trees. HortScience 12:347-348.

Brockerhof, H. 1974. Model of interaction of polar lipids, cholesterol, and proteins in biological membranes. Lipids 9:645-650.

Cossins, A.R. 1983. The adaptation of membrane structure and function to changes in temperature, p. 5-32. In: A.R. Cossins and P. Sheferline (eds.). Cellular acclimation to environmental changes. Cambridge Univ. Press, Cambridge. 
1999

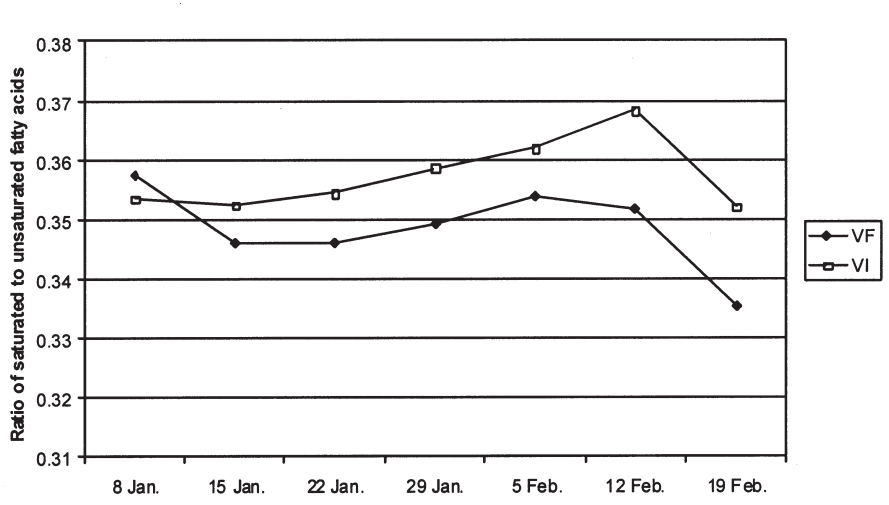

2000

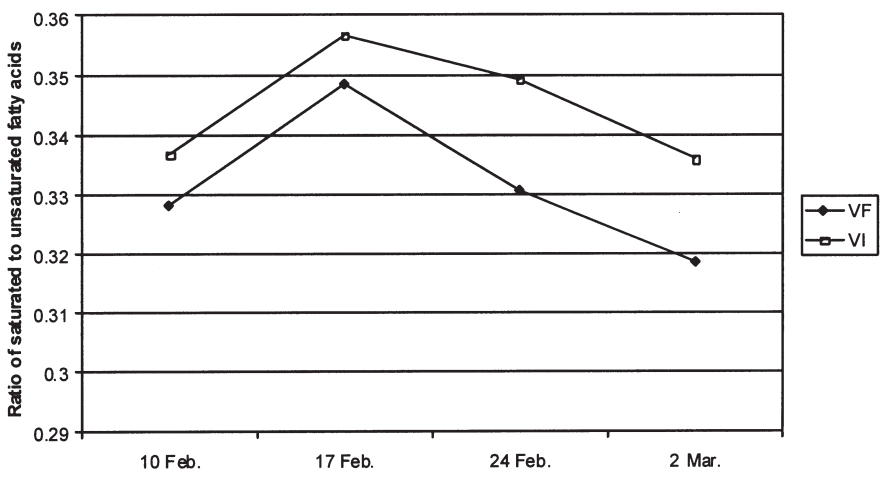

Fig. 5. The ratio of saturated [palmitic (16:0) and stearic (18:0)] to unsaturated fatty acids [oleic (18:1), linoleic (18:2), and linolenic (18:3)] in floral buds of control (VF) and peach latent mosaic viroid (PLMVd)-inoculated (VI) trees. Each data point represents the average of eight samples. Differences in the ratio of saturated to unsaturated fatty acids between the control and VI trees for sample dates after 5 Feb. 1999 and 17 Feb. 2000 were significant $(t$ test $P=0.05$ ).

Diener, T.O. 1987. In: The Viriods. Plenum Press. New York.

Erez, A., S.Y. Wang, and M. Faust. 1997. Lipids in peach buds during dormancy, a possible involvement in dormancy control. Adv. Hort. Sci. 11:128-132.

Faust, M. and S.Y. Wang. 1993. Biochemical events associated with resumption of growth in temperate-zone fruit trees. Acta Hort. 329:257-264.

Gibson, P.G. and G.L. Reighard. 2002. Chilling requirement and postrest heat accumulation in peach trees inoculated with peach latent mosaic viroid. J. Amer. Soc. Hort. Sci. 127:333-336.

Gibson, P.G., G.L. Reighard, S.W. Scott, and D.R. Ouellette. 2001a. Using graft transmissible agents in Y-trained peach systems. Acta Hort. 557:139-144.

Gibson, P.G., G.L. Reighard, S.W. Scott, and M.T. Zimmerman. 2001b. Identification of graft-transmissible agents from 'Ta Tao 5' peach and their effects on 'Coronet' peach. Acta Hort. 550:309-314.

Goad, L.J. 1983. How is sterol synthesis regulated in higher plants? Biochem. Soc. Trans. 11:548-552.

Grossman, Y.L. and T.M. Dejong. 1994. Carbohydrate requirements for dark respiration by peach vegetative organs. Tree Physiology $14: 37-48$

Grunwald, C. 1975. Plant sterols. Annu. Rev. Plant Physiol. 26:209_ 236.

Harris, P. and A.T. James. 1968. The effect of low temperature on fatty acid biosynthesis in plants. Biochem. J. 112:325-330.

Lynch, D.V. and P.L. Steponkus. 1987. Plasma membrane lipid alteration associated with cold acclimation of winter rye seedlings (Secale cereale L. cv. Puma). Plant Physiol. 83:761-767.

Murakami, Y., M. Tsuyama, Y. Kobayashi, H. Kodama, and K. Iba. 2000. Trienoic fatty acids and plant tolerance of high temperature. Science 287:476-479.

Norman, H.A., P. Pillai, and J.B. St. John. 1991. In vitro desaturation of monogalactosyldiacylglycerol and phosphatidylcholine molecular species by chloroplast homogenates. Phytochemistry 30:2317-2322.

Oldfield, E. and D. Chapman. 1972. Dynamics of lipid membranes: Heterogeneity and the role of cholesterol. FEBS Lett. 23:285-297.

Reighard, G.L. 1998. Manipulation of peach phenology, growth, and fruit maturity using interstems. Acta Hort. 465:567-572.

Richardson, E.A., S.D. Seely, D.R. Walker, J.L. Anderson, and G.L. Ashcroft. 1975. Phenoclimatography of spring peach bud development. HortScience 10:236-237.

Shamloul, A.M. 1995. Peach latent mosaic viroid: nucleotide sequence of an Italian isolate, sensitive detection using RT-PCR and geographic distribution. Acta Hort. 386:522-530.

Sikorska, E. and T. Farkas. 1982. Sterols and frost hardening of winter rape. Physiol. Plant 56:349-352.

Siller-Cepeda, J.H.,L.H. Fuchigami, and T.H.H.Chen. 1992a. Glutathione content in peach buds in relation to development and release of rest. Plant Cell Physiol. 33:867-872.

Siller-Cepeda, J.H., L.H. Fuchigami, and T.H.H. Chen. 1992b. Hydrogen cyanamid-induced bud break and phytotoxicity in 'Redhaven' peaches. HortScience 27:874-876.

Spiegel-Roy, P. and F.M. Alston. 1979. Chilling and post-dormant heat requirement as selection criteria for late flowering pears. J. Hort. Sci. 54:115-120.

Sukhija, P.S. and D.L. Palmquist. 1988. Rapid method for determination of total fatty acid content and composition of feedstuffs and feces. J. Agr. Food Chem. 36:1202-1206.

Thompson, G.A. 1979. Molecular control of membrane fluidity, p. 347-363. In: J.M. Lyons, D. Grahas, and J.K. Raison (eds.). Low temperature stress in crop plants. Academic, New York, London.

Wang, S.Y. and M. Faust. 1988. Changes of fatty acids and sterols in apple buds during bud break induced by a plant bioregulator, thidiazuron. Physiol. Plant. 72:115-120.

Wang, S.Y. and M. Faust. 1990. Changes in membrane lipids in apple buds during dormancy and bud break. J. Amer. Soc. Hort. Sci. 115:803-808.

Yoshida, S. 1984. Chemical and biophysical changes in the plasma membrane during cold acclimation of mulberry bark cells (Morus bombycis Koidz. Goroji). Plant Physiol. 76:257-265. 